\title{
Assimilation of the Observational Data in the Marine Ecosystem Adaptive Model at the Known Mean Values of the Processes in the Marine Environment
}

\author{
I. E. Timchenko, I. P. Lazarchuk*, E. M. Igumnova \\ Marine Hydrophysical Institute, Russian Academy of Sciences, Sevastopol, Russian Federation \\ *e-mail: lazarchuk.syst.analysis@mhi-ras.ru
}

\begin{abstract}
Assimilation of observational data in the marine ecosystem adaptive models constructed by the adaptive balance of causes method is considered. It is shown that the feedback balance between the ecosystem variables and the rates of their change used in the method equations, permits to introduce a stationary state of the ecosystem characterized by the observed mean values of the variables. The method for assessing the normalized coefficients of influences based on application of the Euler theorem on homogeneous functions to the functions representing material balances of biochemical reactions of the substance transformation is proposed. It is shown that the normalized ratios of the modeled process mean values can be used as the estimates of the reaction product derivatives obtained on the basis of their resources included in the equations of material balances. Onedimensional adaptive model of the sea upper layer ecosystem is constructed as an example; it is based on the scheme of cause-effect relations of the Fasham, Dacklow and McKelvie model of plankton dynamics and nitrogen cycle It is shown that in such a model, observational data is assimilated by automatic adaptation of the model variables to the assimilated information providing that the substance material balance are preserved in the transformation reactions. The data simulating both observations of the chlorophyll $a$ concentrations and the marine environment dynamics are assimilated in the model. Time scenarios of the biochemical processes are constructed; they confirm applicability of the proposed method for assessing the effect coefficients based on the ratios of the simulated process mean values.
\end{abstract}

Keywords: adaptive balance of causes method, ABC-method, adaptive models, marine ecosystem.

DOI: 10.22449/1573-160X-2017-5-71-86

(c) 2017, I. E. Timchenko, I. P. Lazarchuk*, E. M. Igumnova

(C) 2017, Physical Oceanography

\section{Introduction}

A growing amount of information on the processes in the sea upper layer, which is due to the development of numerical modeling of marine environment physical characteristics [1] and sea surface satellite monitoring [2, 3], offers the prospects of operative control upon the scenarios of those processes which are not directly observed. As the non-observable processes are related to the observed ones by the dependences known from the experience, a general system of equations of the sea environment mathematical model provides the calculation of scenarios of all modeled processes.

In the sea upper layer ecosystems the complex biochemical interactions of substances and living objects [4,5] develop against a background of dynamic processes. The main problem of ecosystem modeling is a parameterization of cause-effect relationships between the model variables: we have to involve a huge number of empirical relationships and coefficients which significantly complicate the model equations and lead to inevitable errors in the modeling results.

Assimilation of observational data in the models of ecosystems is required for making the model estimations of process scenarios more realistic. This purpose is 
provided by probabilistic and variational methods of observational data assimilation in numerical models of ocean dynamics [6-10]. In a series of studies, the equations of substance transport and diffusion are supplemented by the functions of sources and sinks describing the chemical-biological reactions between them. This approach provides the assimilation of the observations of chemical-biological processes directly in the equations of numerical models of the marine environment. However, such approach results in the complex models of marine ecosystems containing the partial differential equation reaction-advectiondiffusion [2, 5, 11].

The task of monitoring the dynamics of processes in marine ecosystems is simplified when the archive observational data (which can be used in simpler marine ecosystem adaptive models [12-15]) are accumulated. In these models, the reactions of chemical and biological substances transformation are described by the coefficients of impacts determined from the observational data. Assimilation of observational data in the adaptive models of ecosystems comes down to automatic adjustment of ecosystem variables to the external impacts (the results of calculations of substance transport and diffusion as well as the results of current observations of biochemical processes are used as them). If the volume of accumulated information is sufficient, a complex system of functions describing the relations between the biochemical processes can be replaced by a simpler system of coefficients of adaptive ecosystem model impacts.

In the present work such simplified approach to the observational data assimilation in the marine ecosystem adaptive model is considered. The purpose of the work was to test a possibility in principal of observation assimilation in the ecosystem adaptive model when there is a minimum volume of a priori information on real biochemical processes in the marine environment. It was assumed that only the signs of the effects of certain processes on other ones and mean values of these processes are known. To carry out the mentioned test, the numerical experiments carried out with the adaptive variant of simplified model of plankton dynamics and nitrogen cycle proposed in were used [16].

Adaptive approach to the modeling of dynamic processes in marine ecosystems. This approach is characterized by the use of balanced feedbacks in the equations of ecosystem model biochemical variables. As is known, the feedbacks make the process variation rate to be dependent on the processes themselves. If the simulated ecosystem variable falls into the expression for the source function in the right side of the equation, then a positive feedback takes place. If the mentioned variable falls into the sink function, there is a negative feedback. Usually the simulated variables are included into the source and sink functions in the right side of the equations of marine environment dynamic model without any additional conditions which balance the positive and negative feedbacks in each model equation. These conditions are replaced by the global balance of negative feedbacks which is characteristic of the traditional models of marine ecosystems.

For instance, in the known nitrogen-based model of plankton dynamics developed by Fasham, Ducklow and McKelvie [16] hydrochemical processes in the mixed upper layer of the sea are represented by seven ecosystem components: the concentrations of phytoplankton $(P)$, zooplankton $(Z)$, bacteria $(B)$, nitrogen 
nitrates $(\mathrm{Nn})$, ammonium nitrogen $(\mathrm{Na})$, dissolved organic nitrogen $(\mathrm{Nd})$ and detritus $(D)$. The scheme of cause-effect relationships based on this model is shown in Fig. 1.

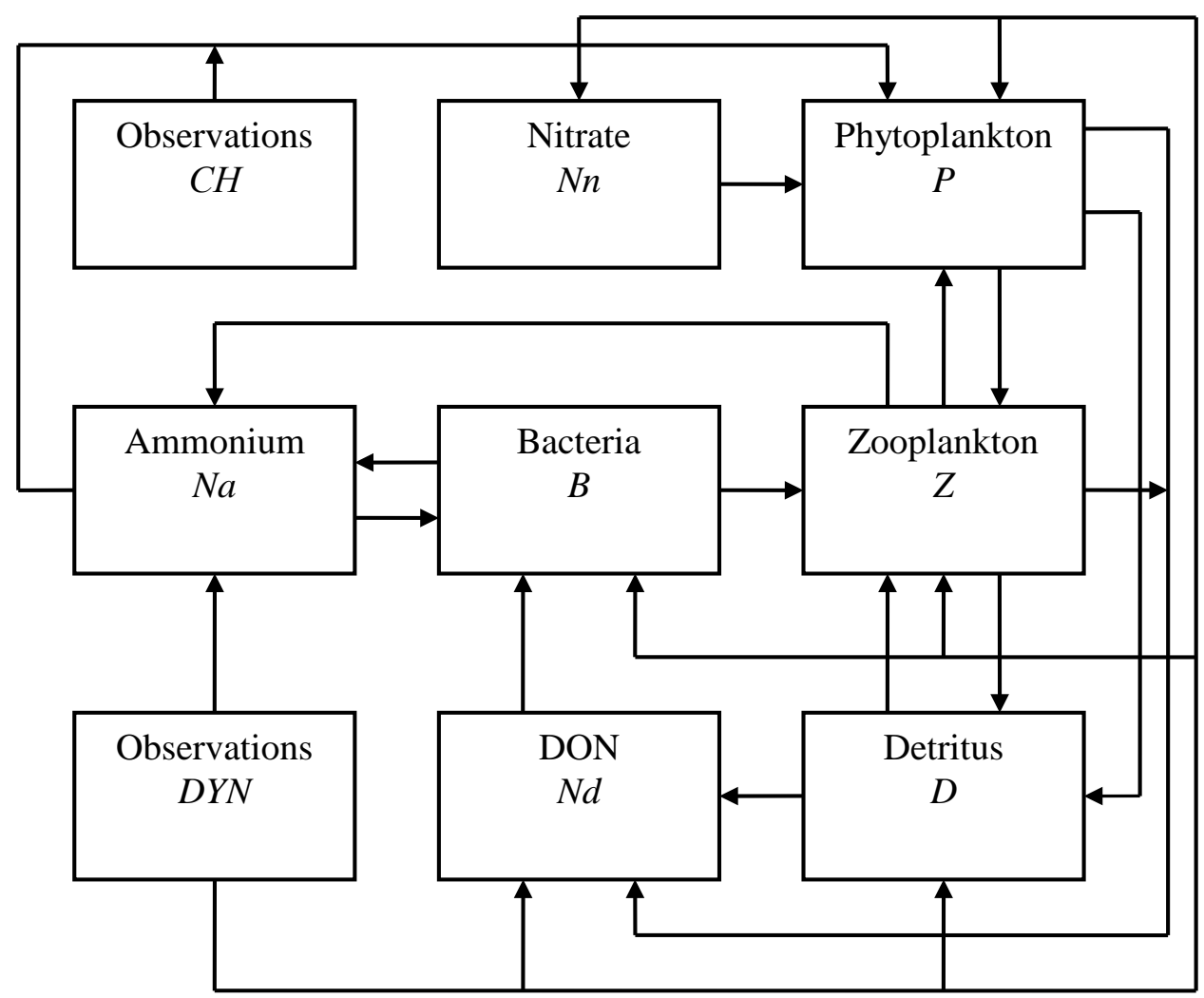

Fig. 1. The scheme of cause-effect relationships between the ecosystem variables based on the model developed by Fasham, Ducklow and McKelvie [16]. CH is the observations of chlorophyll $a$ concentration assimilated in the phytoplankton $P$ block; DYN is the data on marine ecosystem dynamics assimilated in other blocks

Hydrodynamic part of the model describes seasonal variations of the mixed layer depth (the concentration of the modeled substances depends on them). So, in the involvement mode under effect of the increasing near-water wind, the concentration of the simulated substances in the upper layer decreases. This is equivalent to an increase in the sink functions in the corresponding equations of the ecosystem differential model. An inverse phenomenon (the increase in the values of source functions) is observed at the decrease of the mixed layer depth when the concentration of substances increases.

In the considered work the functions taking into account the marine environment dynamics under effect of near-water wind were included into the model of biochemical processes represented by common differential equations. Particularly, the equation for the phytoplankton concentration took the following form in this model [16]: 


$$
\frac{d P}{d t}=\left(1-\gamma_{1}\right) \sigma(t) P-G_{1}-\mu_{1} P-\frac{\left(m+h^{+}(t)\right) P}{M},
$$

where: $\gamma_{1}$ is a share of primary production transformed into dissolved organic matter by the phytoplankton; $\sigma(t)$ is a mean daily specific rate of phytoplankton growth, $G_{1}$ is a rate of phytoplankton consumption by the plankton; $\mu_{1}$ is a specific rate of natural phytoplankton mortality, $m+h^{+}(t)$ is a rate of vertical exchange through the boundary of a homogeneous layer, $h^{+}(t)=\max [h(t), 0]$; $M(t)$ is a mixed layer depth.

Let us consider positive and negative feedbacks in this equation. The first term is a source function which is related to the phytoplankton concentration $P$ variation rate by the first-order positive feedback. The second and the third terms are the sink functions linking the concentration $P$ with the rate of its variation by firstorder negative feedbacks. The last term is an external effect on the phytoplankton concentration which is due to the dynamics of the mixed upper layer thickness.

Positive and negative feedbacks in this equation are not balanced as the coefficients before the function $P$ in the right part of the equation are not linked by some additional relations. Therefore, inaccuracies which arise when setting the balances of source and sink functions (caused by inaccuracies of parameterization of these coefficients) can result in instability of solutions. The same conclusions follow from the analysis of the remaining model equations as in each of them there are both positive and negative feedbacks and there are no additional conditions balancing their effect. Total balance of transformation of chemical and biological resources into the ecosystem products is provided by combining all the equations into a system and by selecting carefully the parameterizations of intra-system relationships.

Using the adaptive approach to the modeling of ecosystems, the balance of feedbacks is automatically set in each equation of the ecosystem model. This allow one to replace, when it is possible, the complex parameterizations of cause-effect dependencies between the processes by the coefficients of effects estimated according to observational data. Thus, the role of a priori information applied in the adaptive models of ecosystems is determined. Moreover, the task of joint description of dynamic and biochemical processes becomes easier as local (within a computational grid cell) adjustment of ecosystem variables to the external effect of substance advection and diffusion is carried out. This adjustment is also automatically controlled by feedbacks which hold the model variables in the predetermined boundaries of their natural variability. The consequence of this fact is a rapid convergence and stability of the algorithms for numerical solution of ecosystem equations.

A method for constructing adaptive models of marine ecosystems. In the present investigation the models of cause-effect relations between the variables of complex system which are formalized by the Adaptive Balance of Causes method (ABC-method) [12] are called the adaptive models. In this method the second- 
order negative feedbacks between the model variables and rates of their variation contained in each modular equation of the method are applied.

Let for the description of the processes in an ecosystem a variety of interrelated processes represented by $0 \leq u_{i} \leq 2 C_{i}$ functions (which meet the purpose of modeling), where $C_{i}$ - average values of the processes, is used. $u_{i}$ variables have the intra-system effects on each other in accordance with the cause-effect relations which are assumed to be known. These relations form the material balances of the modeled substances which can be represented by the following correlation:

$$
u_{i}=\sum_{j=1, j \neq i}^{n} a_{i j} u_{j}+A_{i},
$$

where $a_{i j}$ are the coefficients of intra-system effects; $n$ is a number of variables; $A_{i}$ are the external effects brought against the ecosystem. Intra-system effects express the reactions of $u_{j}$ resources transformation into $u_{i}$ products. As a result of these reactions, the amount of product is incremented or decreased by the number of units that is provided by the available quantity of resources and the conditions for the reaction.

$A B C$-method is based on the following assumptions:

1. There is a stationary state of the ecosystem equilibrium in which, in the absence of external effects, the ecosystem model variables in the correlation (1) take their mean values:

$$
C_{i}=\sum_{j=1, j \neq i}^{n} a_{i j} C_{j} .
$$

$\left\{C_{i}\right\}$ stationary state of $\left\{u_{i}\right\}$ adaptive model of processes system is determined by setting the values of $\left\{a_{i j}\right\}$ effect coefficients. These values remain when averaging the expressions (1).

2. External effects on the ecosystem do not change its stationary state; they just result in deviations of $u_{i}$ variables from their $C_{i}$ mean values.

3. Ecosystem variables adapt to the external effects in such a way that the material balances of intra-system $\left(\sum_{j=1, j \neq i}^{n} a_{i j} u_{j}\right)$ and external $\left(A_{i}\right)$ reactions of substance interaction remain in the form of increments in number of reaction products in reference to their mean values $C_{i}$ :

$$
u_{i}=C_{i}+\sum_{j=1, j \neq i}^{n} a_{i j} u_{j}+A_{i} .
$$

Ecosystem variables continuously adapt to external effects, adapting to them within their own variability intervals $0 \leq u_{i} \leq 2 C_{i}$. 
The introduced assumptions mean that the ecosystem is resistant to external influences $A_{i}$ and returns to its stationary state whenever external effects disappear. Consequently, the applicability of the $A B C$-method is restricted by the case when the ecosystem is stable and external effects do not change its structure.

In [12-15] it was shown that the system of equations of the Adaptive Balance of Causes method is constructed in such a way as to maintain the dynamic balances of the modeled substance masses (1). For this purpose the following general (modular) structure of equations is proposed:

$$
\frac{d u_{i}}{d t}=r_{i} u_{i}\left[F_{i}^{-}-F_{i}^{+}\right]
$$

where $r_{i}$ are specific rates of variable change; $F_{i}^{-}$is a monotonously decreasing and $F_{i}^{+}-$a monotonously increasing basic functionals. Their arguments are the value differences of the left and the right parts of the dynamic balance equations (1). The functionals are connected by additional conditions: $F_{i}^{-}+F_{i}^{+}=2 C_{i}$, which balance feedbacks in the equations of the model (4) taking into account the resource limitation of intra-system reactions $u_{i} \leq 2 C_{i}$. Particularly, $F_{i}^{+}$functionals can be determined in the following way:

$$
F_{i}^{+}=F_{i}^{+}\left(u_{i}-\sum_{j=1, j \neq i}^{n} a_{i j} u_{j}-A_{i}\right)=u_{i}-\sum_{j=1, j \neq i}^{n} a_{i j} u_{j}-A_{i} .
$$

Then the following logistic modular equation system of the adaptive model takes place:

$$
\frac{d u_{i}}{d t}=2 r_{i} u_{i}\left[C_{i}-\left(u_{i}-\sum_{j=1, j \neq i}^{n} a_{i j} u_{j}-A_{i}\right)\right],(i, j=1,2, \ldots, n) .
$$

It is easy to see that non-trivial solutions of the system of equations (5) satisfy the condition (3).

In order to make intra-system and external effects not to output model variables beyond the areas of their variation $0 \leq u_{i} \leq 2 C_{i}$, the following additional restrictions are imposed:

$$
\begin{gathered}
\left|\sum_{j=1, j \neq i}^{n} a_{i j} u_{j}\right| \leq C_{i}, \\
u_{i}=\operatorname{IF}\left[u_{i}<0 ; 0 ; \quad I F\left(u_{i}>2 C_{i} ; 2 C_{i} ; u_{i}\right)\right] .
\end{gathered}
$$

Negative second-order feedbacks stabilize the numerical solution of the system of equations (5). In its turn, this provides a rapid adaptation of model variable values to each other and to external effects.

The main problem for practical implementation of marine ecosystem adaptive models is a determination of the coefficients of $a_{i j}$ effects according to the observation data. Up to date, a series of approaches for solving this problem have 
been developed [12-15]. In the present investigation we will focus on the method for assessing the effect coefficients based on the assumption that they retain their values during the averaging of balance relations (1) (i. e. when the ecosystem shifts to the stationary state) as $a_{i j}$ coefficients show which part of $u_{j}$ resources convert into $u_{i}$ products.

Assimilation of observational data by assessing the coefficients of effects according to mean values of ecosystem variables. Observational data of biochemical processes and computational data on marine environment dynamics are the external effect factors in relation to the adaptive models of marine ecosystems. The assimilation containing in the data observations means the adaptation of model variables to the external effects which are directly included into the corresponding equations of the adaptive model (5) as the additional functions of $A_{i}$ sources and sinks. Therefore, the assimilation problem consists in the initialization of the effect coefficients on the basis of that information on the modeled processes which is contained in the observations.

If the values of the variables are known, then the hypothesis about the homogeneity of functions representing the material balances of intra-system reactions (1) can be used for assessing the effect coefficients in the system of equations (5). The Euler theorem on the homogeneous functions [17] can be also applied to the abovementioned material balances. According to this theorem, if $t$ is a certain parameter and the following equality takes place

$$
\sum_{i=1}^{n} u_{i}\left(t u_{j}\right)=t \sum_{i=1}^{n} u_{i}\left(u_{j}\right)
$$

then $u_{i}=\sum_{j=1}^{n} \frac{\partial u_{i}}{\partial u_{j}} u_{j}$

In this case, $\partial u_{i} / \partial u_{j}$ partial derivatives can be used as $a_{i j}$ coefficients of effects in the $A B C$-model of system (5) and the system of equations takes the following general form:

$$
\frac{d u_{i}}{d t}=2 u_{i}\left[C_{i}-\left(u_{i}-\sum_{j=1, j \neq i}^{n} \frac{\partial u_{i}}{\partial u_{j}} u_{j}-A_{i}\right)\right],(i, j=1,2, \ldots, n) .
$$

If there are archived observations of $\bar{u}_{i}$ and $\bar{u}_{j}$ averaged processes, then the values of $\partial \bar{u}_{i} / \partial \bar{u}_{j}$ relations are determined by the scenarios of these processes averaged with a certain scale. In the considered case, when only mean values of $C_{i}$ processes are assumed to be known, approximate estimates can be used as the effect coefficients in the system of equations (5). The relation of the observed mean values $C_{i} / C_{j}$ characterizes the mean rate of $u_{i}$ reaction products in relation to $u_{j}$ resources. Therefore, in the absence of external effects the balance correlation (3) can be expressed in the following form: 


$$
u_{i}=C_{i}+\sum_{j=1, j \neq i}^{n} a_{i j}^{\prime} \frac{C_{i}}{C_{j}} u_{j}
$$

In the formula (8) $a_{i j}^{\prime}$ coefficients are the dimensionless quantities and $C_{i} / C_{j}$ relation of mean values plays a role of $\partial u_{i} / \partial u_{j}$ derivatives. In order to exclude the violation of solution stability of equation system (5) at such an approximation of intrasystem effects, the normalization of $a_{i j}^{\prime}$ coefficients should be carried out. According to the conditions (6)-(7), the stability of solutions will be guaranteed if each sum of positive and negative effect coefficients does not exceed 0.5. We assume that in the equation for $u_{i}$ variable of an ecosystem model $m$ of positive and $n-m$ of negative effects of $u_{j}$ resources are contained. Then it follows from the inequality (6) that the coefficients of positive $a_{i j}^{+}$and negative $a_{i j}^{-}$ effects in the system of equations (5) should satisfy the following conditions:

$$
a_{i j}^{+} \leq \frac{1}{2 m},(1 \leq j \leq m) ; \quad a_{i j}^{-} \leq \frac{1}{2(n-m)},(m+1 \leq j \leq n) .
$$

Hence, for the case when there is $m$ of positive and $n-m$ of negative effects, the balance correlation (8) can be represented as follows:

$$
u_{i}=C_{i}+\frac{C_{i}}{2 m} \sum_{j=1, j \neq i}^{m} C_{j}^{-1} u_{j}-\frac{C_{i}}{2(n-m)} \sum_{j=m+1, j \neq i}^{n} C_{j}^{-1} u_{j} .
$$

Taking into account the expression (10), the system of equations of $A B C$-model (5) takes the following form:

$$
\frac{d u_{i}}{d t}=2 r_{i} u_{i}\left\{C_{i}-\left[u_{i}-\frac{C_{i}}{2 m} \sum_{j=1, j \neq i}^{m} C_{j}^{-1} u_{j}+\frac{C_{i}}{2(n-m)} \sum_{j=m+1, j \neq i}^{n} C_{j}^{-1} u_{j}-A_{i}\right]\right\} .
$$

In order to take into account the resource limitations of growth processes (for example, in the group of $A B C$-model processes of $\left\{u_{p}\right\}(m+1 \leq p \leq s)$ ecosystem) it is necessary to use $A G_{i} \arg \min \left(a_{i m+1} u_{m+1}, \ldots, a_{i s} u_{s}\right)$ logical operators [3; 12] in the equation (11):

$$
\begin{gathered}
\frac{d u_{i}}{d t}=2 r_{i} u_{i}\left\{C_{i}-\left[u_{i}-\frac{C_{i}}{2 m} \sum_{j=1, j \neq i}^{m} C_{j}^{-1} u_{j}-\right.\right. \\
\left.\left.-A G_{i} \arg \min \left(a_{i m+1} u_{m+1}, \ldots, a_{i m+s} u_{m+s}\right)+\frac{C_{i}}{2(n-s-1)} \sum_{j=s+1, j \neq i}^{n} C_{j}^{-1} u_{j}-A_{i}\right]\right\}, \\
A G_{i} \arg \min \left(a_{i m+1} u_{m+1}, \ldots, a_{i s} u_{s}\right)=\operatorname{IF}\left(a_{i p} u_{p}=M_{p} ; a_{i p} u_{p} ; 0\right), \\
M_{p}=\min \left(a_{i m+1} u_{m+1}, \ldots, a_{i s} u_{s}\right) ; \\
a_{i p}=\frac{C_{i}}{2 s} C_{p}^{-1} .
\end{gathered}
$$


If we consider temporal scenarios of the ecosystem variables at a single marine environment point at $t_{k}$ discrete time moments separated by $\Delta t=t_{k+1}-t_{k}$ intervals, then the system of equations of the ecosystem (5) $A B C$-model can be represented in finite differences according to the Euler method:

$$
\begin{aligned}
& u_{i}^{k+1}=u_{i}^{k}+2 \Delta t r_{i} u_{i}^{k}\left[C_{i}-\left(u_{i}^{k}-\sum_{j=1, j \neq i}^{n} a_{i j} u_{j}^{k}-A_{i}^{k}\right)\right]= \\
& =u_{i}^{k}+2 \Delta t r_{i} u_{i}^{k} C_{i}-2 \Delta t t_{i} u_{i}^{k}\left(u_{i}^{k}-\sum_{j=1, j \neq i}^{n} a_{i j} u_{j}^{k}-A_{i}^{k}\right) .
\end{aligned}
$$

For these difference equations it is convenient to take an additional $2 \Delta t r_{i} C_{i}=1$ condition. Then the equation (12) is simplified:

$$
u_{i}^{k+1}=2 u_{i}^{k}\left[1-\frac{1}{2 C_{i}}\left(u_{i}^{k}-\sum_{j=1, j \neq i}^{n} a_{i j} u_{j}^{k}-A_{i}^{k}\right)\right] .
$$

Thus, the suggested method for observational data assimilation comes down to the inclusion of $A_{i}$ observed values of external effects reduced to $\left(-C_{i} \leq A_{i} \leq C_{i}\right)$ intervals into the adaptive model equations for $u_{i}$ variables. Due to the errors of the effect coefficients estimated by the relations of mean values with the normalization according to the formulas (9), finite difference equations (13) will result in deviations of process scenarios from some (conditional) $C_{i}^{*}$ mean values which differ from the observed $C_{i}$ mean values. However, it is known from the considered properties of the $A B C$-method that the external effects (that have zero mean values) do not change stationary state of the system of equations of $\left\{C_{i}\right\}$ model. Therefore, it is easy to make the adjustments eliminating the difference between actual and conditional mean values in the calculated process scenarios in the ecosystem.

Assimilation of observational data in the adaptive version of the marine ecosystem model. As an example of the proposed method application, we are to consider the assimilation of observational data in the adaptive version of marine ecosystem model based on the simplified scheme of model [16] cause-effect relations shown in Fig. 1. Substituting the corresponding functions of effects in the system of equations (5), for the system of $A B C$-equations of this model we obtain:

$$
\begin{gathered}
\frac{d P}{d t}=2 r_{P} P\left\{C_{P}-\left[P-a_{P / N_{a}} N_{a}-a_{P / N_{n}} N_{n}+a_{P / Z} Z-F(C H)-D Y N(P)\right]\right\}, \\
\frac{d Z}{d t}=2 r_{Z} Z\left\{C_{Z}-\left[Z-a_{Z / B} B-a_{Z / D} D-a_{Z / P} P-D Y N(Z)\right]\right\}, \\
\frac{d B}{d t}=2 r_{B} B\left\{C_{B}-\left[B-a_{B / N_{a}} N_{a}-a_{B / N_{d}} N_{d}-D Y N(B)\right]\right\} \\
\frac{d D}{d t}=2 r_{D} D\left\{C_{D}-\left[D-a_{D / P} P-a_{D / Z} Z-D Y N(D)\right]\right\}
\end{gathered}
$$




$$
\begin{gathered}
\frac{d N_{a}}{d t}=2 r_{N_{a}} N_{a}\left\{C_{N_{a}}-\left[N_{a}+a_{N_{a} / P} P+a_{N_{a} / B} B-a_{N_{a} / Z} Z-D Y N\left(N_{a}\right)\right]\right\}, \\
\frac{d N_{n}}{d t}=2 r_{N_{n}} N_{n}\left\{C_{N_{n}}-\left[N_{n}+a_{N_{n} / P} P-D Y N\left(N_{n}\right)\right]\right\}, \\
\frac{d N_{d}}{d t}=2 r_{N_{d}} N_{d}\left\{C_{N_{d}}-\left[N_{d}-a_{N_{d} / P} P-a_{N_{d} / Z} Z-a_{N_{d} / D} D+a_{N_{d} / B} B-D Y N\left(N_{d}\right)\right]\right\} .
\end{gathered}
$$

The external effects of the sea upper mixed layer depth dynamics on the model variables are denoted by $\operatorname{DYN}\left(u_{i}\right)$ symbols in the equations (14), by $F(C H)$ - the effect of chlorophyll $a$ observations (assimilated in the equation for $P$ ) on the phytoplankton concentration. The braces in the right parts of adaptive models (14) retain the material balances of ecosystem component interaction biochemical reactions provided by feedback balances in each equation with regard to internal effects.

We assume that a priori there is minimal information on the ecosystem: only mean values of $C_{i}$ model variables and the signs of intrasystem effects are known. In these conditions it is necessary to use the abovementioned approach for constructing the estimates of coefficients by the Euler theorem in the assumption about the homogeneity of balance correlations (2). To determine $a_{M / N}$ coefficients of the effects, we use formulas (8) and (9) in which we substitute the known mean values of the corresponding ecosystem variables.

Let us write down the model (14) in finite differences using the modular equations of the $A B C$-method (12):

$$
\begin{gathered}
P^{k+1}=2 P^{k}\left\{1-\frac{1}{2 C_{P}}\left[P^{k}-a_{P / N_{a}} N_{a}^{k}-a_{P / N_{n}} N_{n}^{k}+a_{P / Z} Z^{k}-F(C H)-D Y N(P)\right]\right\}, \\
Z^{k+1}=2 Z^{k}\left\{1-\frac{1}{2 C_{Z}}\left[Z^{k}-a_{Z / B} B^{k}-a_{Z / D} D^{k}-a_{Z / P} P^{k}-D Y N(Z)\right]\right\}, \\
B^{k+1}=2 B^{k}\left\{1-\frac{1}{2 C_{B}}\left[B^{k}-a_{B / N_{a}} N_{a}^{k}-a_{B / N_{d}} N_{d}^{k}-D Y N(B)\right]\right\}, \\
D^{k+1}=2 D^{k}\left\{1-\frac{1}{2 C_{D}}\left[D^{k}-a_{D / P} P^{k}-a_{D / Z} Z^{k}-D Y N(D)\right]\right\}, \\
N_{a}^{k+1}=2 N_{a}^{k}\left\{1-\frac{1}{2 C_{N_{a}}}\left[N_{a}^{k}+a_{N_{a} / P} P^{k}+a_{N_{a} / B} B^{k}-a_{N_{a} / Z} Z^{k}-D Y N\left(N_{a}\right)\right]\right\}, \\
N_{n}^{k+1}=2 N_{n}^{k}\left\{1-\frac{1}{2 C_{N_{a}}}\left[N_{n}^{k}+a_{N_{n} / P} P^{k}-D Y N\left(N_{n}\right)\right]\right\}, \\
N_{d}^{k+1}=2 N_{d}^{k}\left\{1-\frac{1}{2 C_{N_{d}}}\left[N_{d}^{k}-a_{N_{d} / P} P^{k}-a_{N_{d} / Z} Z^{k}-a_{N_{d} / D} D^{k}+a_{N_{d} / B} B^{k}-D Y N\left(N_{d}\right)\right]\right\} .
\end{gathered}
$$

In order to provide the stability of the system of equations (15) solution, we supplement it with the conditions (7):

$$
u_{i}^{k}=I F\left[u_{i}^{k}<0 ; 0 ; \operatorname{IF}\left(u_{i}^{k}>2 C_{i} ; 2 C_{i} ; u_{i}^{k}\right)\right] .
$$




\section{Computational experiments on the observational data assimilation in the ecosystem model}

Let us consider simulated computational experiments with the constructed adaptive model. As the purpose of the experiments was to test the supposed method for observational data assimilation, it was carried out without reference of the ecosystem adaptive model (15) to the specific marine water area. Therefore, $C_{i}$ values in $\mathrm{mg} / \mathrm{m}^{3}$ (given in Table 2 as the elements of the matrix of $a_{i j}$ intrasystem effects) were chosen as mean values of the model variables. These mean values were used to estimate the absolute values of normalized effect coefficients which are represented in Table 2 as the elements of the matrix of $a_{i j}$ intrasystem effects.

Table 1

Mean $\left(C_{i}\right)$ and conditional mean $\left(C_{i}^{*}\right)$ values of marine ecosystem components

\begin{tabular}{c|c||c|c}
\hline Mean values & $C_{i}$ & Conditional mean values & $C_{i}^{*}$ \\
\hline$C_{P}$ & 4.00 & $C_{P}^{*}$ & 3.00 \\
$C_{Z}$ & 4.20 & $C_{Z}^{*}$ & 5.97 \\
$C_{B}$ & 3.74 & $C_{B}^{*}$ & 2.06 \\
$C_{D}$ & 4.40 & $C_{D}^{*}$ & 6.64 \\
$C_{N_{a}}$ & 4.76 & $C_{N_{a}}^{*}$ & 7.13 \\
$C_{N_{n}}$ & 4.83 & $C_{N_{n}}^{*}$ & 5.58 \\
$C_{N_{d}}$ & 2.92 & $C_{N_{d}}^{*}$ & 3.71 \\
\hline
\end{tabular}

Table 2

Coefficients of marine ecosystem mutual effects

\begin{tabular}{c|ccccccc}
\hline$a_{i j}$ & $P$ & $Z$ & $B$ & $D$ & $N_{a}$ & $N_{n}$ & $N_{d}$ \\
\hline$P$ & 1.00 & 0.68 & - & - & 0.21 & 0.21 & - \\
$Z$ & 0.17 & 1.00 & 0.18 & 0.15 & - & - & - \\
$B$ & - & 0.45 & 1.00 & - & 0.19 & - & 0.31 \\
$D$ & 0.25 & 0.24 & - & 1.00 & - & - & - \\
$N_{a}$ & - & 0.50 & 0.60 & - & 1.00 & - & - \\
$N_{n}$ & 0.60 & - & - & - & 0.80 & 1 & - \\
$N_{d}$ & 0.12 & 0.12 & 0.39 & 0.11 & - & - & 1.00 \\
\hline
\end{tabular}

As the found coefficients of effects were the approximate estimations of true coefficients, conditional stationary mean values of $C_{i}^{*}$ ecosystem variables should correspond to them. To calculate these coefficients the system of equations (15) was solved with $a_{i j}$ coefficients form Table 2 under arbitrary initial conditions and in the absence of external effects on the ecosystem, i. e. at $F(C H)=D Y N\left(u_{i}\right)=0$. 
In Fig. $2 a, b$ the graphs of integration convergence to conditional stationary solution are given and the calculated conditional mean values of model variables $\left(C_{i}^{*}\right)$ are placed into the Table 2 .
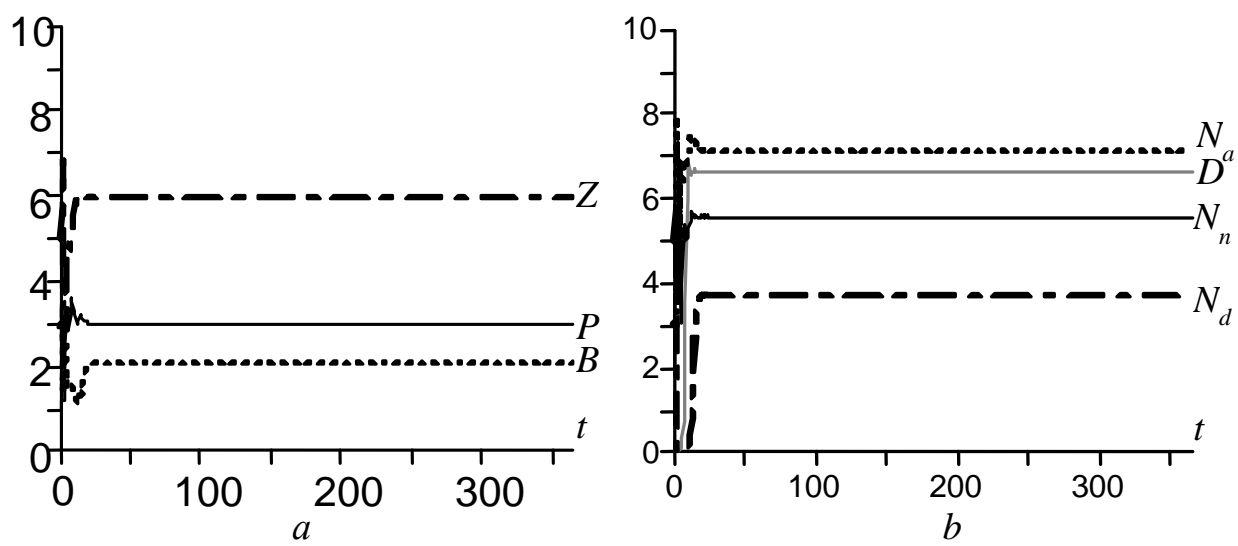

Fig. 2. Convergence of the equation (15) solutions to the conditional stationary state $\left\{C_{i}^{*}\right\}$ from arbitrary initial conditions at normalized effect coefficients determined by the formulas (9)

As it follows from Fig. 2, the convergence of iteration process of $A B C$-model equation (15) solution turned out to be quite rapid. It is obvious from the comparison of $C_{i}$ and $C_{i}^{*}$ (Table 1) values that their values noticeably differ from each other. We recall that the idea of proposed method consisted in the fact that due to the features of the $A B C$-method modeling the mentioned differences between $C_{i}$ and $C_{i}^{*}$ mean values remain constant when including the variables of external effects on the ecosystem with zero mean values. In other words, the reactions of ecosystem adaptive model (15) on the external effects (reduced to zero mean values) consist in deviations of ecosystem variable scenarios from the unchanged conditional mean values. Consequently, in order to obtain true scenarios of variables, it is sufficient just to make the known corrections to the mean values of scenarios calculated according to the model (15).

For testing the aforementioned idea, the observational data of chlorophyll $a$ $(\mathrm{CH})$ concentration deviation from $C_{C H}$ mean values (Fig. 3) were simulated. These data were used as an additional source function on the right part of an equation for the model (15) phytoplankton concentration:

$$
P^{k+1}=2 P^{k}\left\{1-\frac{1}{2 C_{P}}\left[P^{k}-a_{P / N_{a}} N_{a}^{k}-a_{P / N_{n}} N_{n}^{k}+a_{P / Z} Z^{k}-C H\right]\right\} .
$$

The results of observational data assimilation (in the model) of chlorophyll $a$ $\mathrm{CH}$ concentration deviation from $\mathrm{C}_{\mathrm{CH}}^{*}$ mean values are shown in Fig. 4. As expected, these observations had the greatest effect on the scenario of phytoplankton concentrations. 


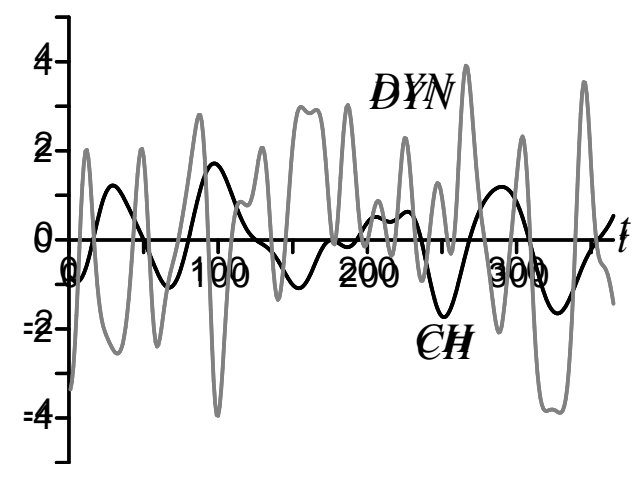

Fig. 3. External effects on the ecosystem: $\mathrm{CH}$ - the data of chlorophyll a concentration deviation from $C_{C H}$ mean values assimilated in the model; $D Y N$ - assimilated computational data on the marine environment dynamics
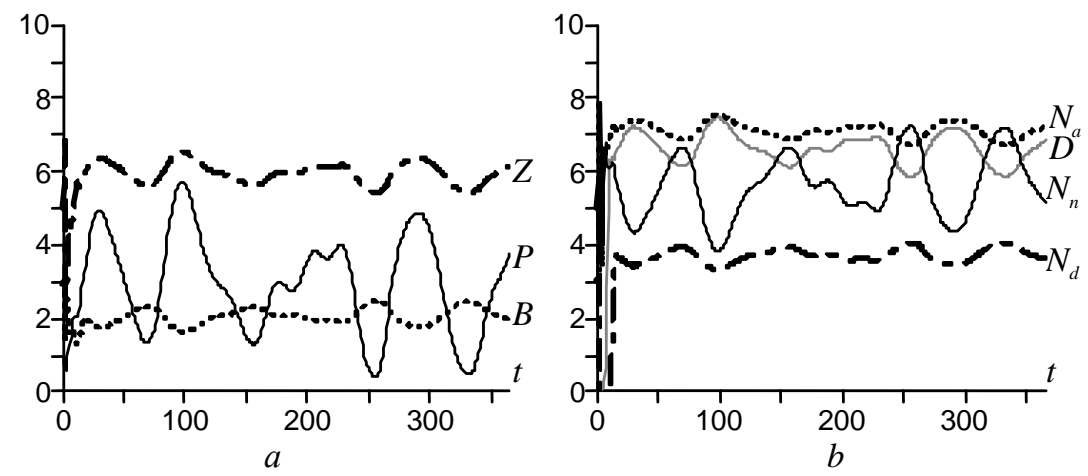

Fig. 4. The results of chlorophyll $a(\mathrm{CH})$ concentration observational data assimilation in the model

This effect has extended to other variables of ecosystem model through the intrasystem cause-effect relations.

In the next computational experiment a simultaneous assimilation of chlorophyll $a$ concentration observational data and the information about marine environment dynamics effect on the intrasystem processes in the ecosystem was simulated. It was assumed that there are the results of calculations of modeled substance transport and diffusion processes (carried out using a numerical hydrodynamic model) for the marine environment point in which the concentration variation scenarios of these substances are calculated. It was decided that DYN function (Fig. 3) imitates the scenario of marine environment dynamics effect and the concentrations of all modeled substances adapt to it. The results of these data assimilation in the ecosystem model relative to $C_{i}^{*}$ conditional mean values are shown in Fig. 5.

Process scenarios given in Fig. 5 are obtained as deviations from $C_{i}^{*}$ conditional mean values. However, having true mean values of $C_{i}$ variables placed in Table 1 , it is easy to introduce constant corrections to $C_{i}^{*}$ values for obtaining the estimations of true process scenarios in the ecosystem. After introducing these corrections the scenarios take the form shown in Fig. 6 . 

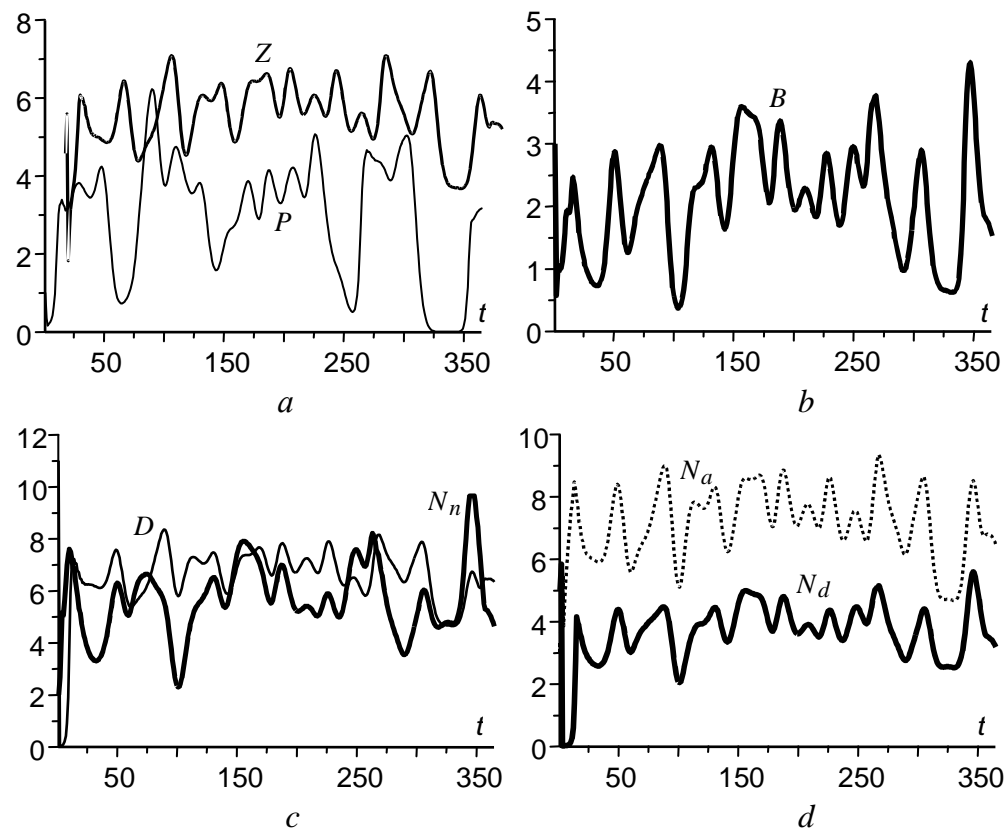

Fig. 5. The results of assimilation in the ecosystem model of chlorophyll $a \mathrm{CH}$ concentration observational data and computational data on $D Y N$ marine environment dynamics in relation to $C_{i}^{*}$ conditional mean values
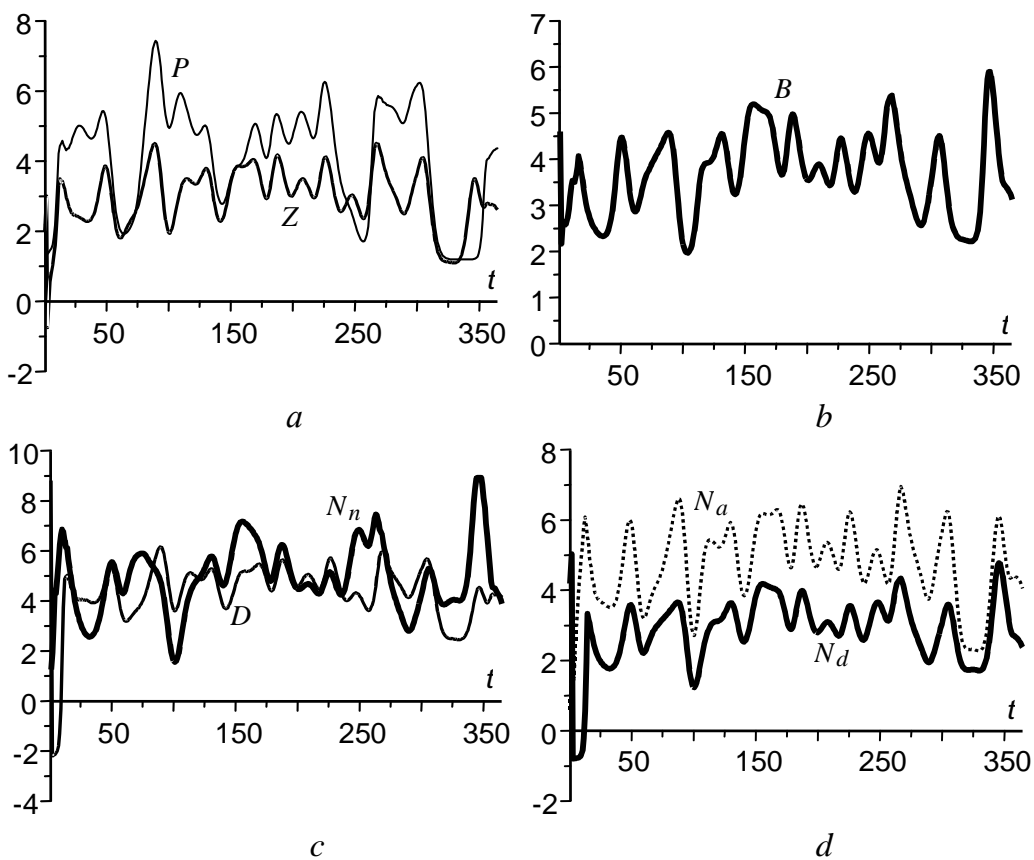

Fig. 6. The results of assimilation in the ecosystem model of chlorophyll $a \mathrm{CH}$ concentration observational data and computational data on DYN marine environment dynamics after reducing them to $C_{i}^{*}$ mean values known from the observations 


\section{Conclusion}

The main conclusion from the performed computational experiments consists in the fact that properties of marine ecosystem adaptive models allow one to propose approximate methods for assimilation of observational data and computational one on the modeled substance transport and diffusion in them. Particularly, estimation of effect coefficients and construction of chemical substance and living organisms' concentration scenarios according to them become possible with limited a priori information contained in mean values of variables and in the known signs of cause-effect relations between them. When assimilating observation time series with zero mean values, model variables adapt to each other and to external effects saving their own mean values. This provides a possibility to reduce the scenarios reproduced by the model to true mean values and obtain realistic scenario estimations which take into account intrasystem cause-effect relations.

The performed numerical experiment confirmed the possibility of using the Euler theorem on homogeneous functions for representing the effect coefficients in the form of partial derivatives of biochemical reaction products (these reactions take place in the ecosystem) by the resources forming their values. The hypothesis on the replacement of these derivatives by the relations of process observed mean values is introduced in the assumption that the coefficients of resource transformation into the products also remain for averaged processes. As a result of derivative replacement by the relations of observed mean values, the coefficients ahead of mean value relations become dimensionless which, in its turn, allow one to introduce their normalization providing the stability of model equation solutions. The method for observational data assimilation has its own specifics because in the marine ecosystem adaptive models an automatic adjustment of ecosystem model variables to the assimilating information takes place. This feature of models does not exclude the following validation of model scenarios by comparing them with current observational data and modeling error correction by other assimilation methods.

Acknowledgements. The study was carried out during the planned investigations carried out by FSBSI MHI of RAS on the project No. 007-00694-16 PR “Fundamental oceanography”.

\section{REFERENCES}

1. Sarkisyan, A.S., 2016. Main Directions in the Simulation of Physical Characteristics of the World Ocean and Seas. Izvestiya, Atmospheric and Oceanic Physics, [e-journal] 52(4), pp. 335-340. doi:10.1134/S0001433816040101

2. Korotaev, G.K. and Eremeev, V.N., 2006. Vvedenie v Operativnuyu Okeanografiyu Chernogo Morya [Introduction to the Operational Oceanography of the Black Sea]. Sevastopol: ECOSIGidrofizika, 382 p. (in Russian).

3. Kochergin, V.P. and Timchenko, I.E., 1987. Monitoring Gidrofizicheskikh Poley Okeana [Monitoring of Hydrophysical Ocean Fields]. Leningrad: Gidrometeoizdat, 280 p. (in Russian).

4. $\quad$ Platt, T. ed., 1984. Matematicheskie Modeli v Biologicheskoy Okeanografii [Mathematical Models in Biological Oceanography]. Paris: UNESCO, 158 p. (in Russian). 
5. Murray, J.D., 2008. Mathematical Biology. II: Spatial Models and Biomedical Applications. New-York: Springer, 736 p.

6. Timchenko, I.E., 1984. Stochastic Modeling of Ocean Dynamics. Chur-London-Paris-NewYork: Harwood Academic Publishers, 320 p.

7. Ghil, M. and Malanotte-Rizzoli, P., 1991. Data Assimilation in Meteorology and Oceanography. In: R.R. Dmowska and B. Saltzman, eds., 1991. Advances in Geophysics. San Diego: Academic Press. Inc. Vol. 33, pp. 141-266. doi:10.1016/S0065-2687(08)60442-2

8. Marchuk, G.I. and Zalesny, V.B., 1993. A Numerical Technique for Geophysical Data Assimilation Problems Using Pontryagin's Principle and Splitting-up Method. Russ. J. Numer. Anal. Math. Modelling, [e-journal] 8(4), pp. 311-326. doi:10.1515/rnam.1993.8.4.311

9. Robinson, A.R. and Lermusiaux, P.F.J., 2000. Overview of Data Assimilation. Harvard Reports in Physical/Interdisciplinary (Ocean Science). Cambridge, Massachusetts: Harvard University, 28 p. Available at: http://robinson.seas.harvard.edu/PAPERS/red_report_62.html [Accessed: 07 July 2017].

10. Mizyuk, A.I., Knysh, V.V., Kubryakov, A.I. and Korotaev, G.K., 2009. Assimilation of the Climatic Hydrological Data in the $\sigma$-Coordinate Model of the Black Sea by the Algorithm of Adaptive Statistics. Physical Oceanography, [e-journal] 19 (6), pp. 339-357. doi:10.1007/s11110-010-9058-2

11. Cantrell, R.S. and Cosner, C., 2004. Spatial Ecology via Reaction-Diffusion Equations. Chichester, UK: John Wiley and Sons, 421 p. doi:10.1002/0470871296.fmatter

12. Timchenko, I.E., Igumnova, E.M. and Timchenko, I.I., 2000. Sistemnyj Menedzhment i ABCTehnologii Ustojchivogo Razvitija [System Management and ABC-Technologies of Sustainable Development]. Sevastopol: ECOSI-Gidrofizika, 225 p. (in Russian).

13. Timchenko, I.E., Igumnova, E.M., Lazarchuk, I.P. and Solodova, S.M., 2014. Obratnye Svjazi v Adaptivnyh Modeljah Morskih Ekosistem [Feedbacks in Adaptive Models of Marine Ecosystems]. Morskoj Gidrofizicheskij Zhurnal, (2), pp. 3-21 (in Russian).

14. Timchenko, I.E., Igumnova, E.M. and Timchenko, I.I., 2016. Adaptive Balance Models for Environmental-Economic Systems. [e-book] CreateSpace Independent Publishing Platform, 486 p. Available at: www.amazon.com/dp/1530208831 [Accessed: 07 July 2017].

15. Ivanov, V.A., Igumnova, E.M. and Timchenko, I.E., 2012. Coastal Zone Resources Management. Kyiv: Akademperiodika, 304 p.

16. Fasham, M., Ducklow, H. and McKelvie, S., 1990. A Nitrogen-Based Model of Plankton Dynamics in the Oceanic Mixed Layer. J. Mar. Res., [e-journal] 48 (3), pp. 591-639. Available at: http://www.ingentaconnect.com/content/jmr/jmr/1990/00000048/00000003/art00006 [Accessed: 07 July 2017].

17. Iljin, V.A. and Poznjak, V.G., 2005. Osnovy Matematicheskogo Analiza. V 2-h Chastjah. Chast 1. [Basic Mathematical Analysis. In 2 Parts. Part 1]. Moscow: Fizmatlit, 648 p. (in Russian). 